(Aus dem II. anatomischen Institut der kgl. Universität Berlin.)

Die Blutkörperchen im bebrüteten Hühnerei. Von

Dr. med. C. S. Engel.

Hierzu Tafel XVII.

Die Frage nach dem Orte der Entstehung des Blutes im bebritteten Hühnerei ist auf Grund zahlreicher Untersuchungen, die über diesen Gegenstand angestellt sind, in der Weise zu beantworten, dass die ersten Blutkörperchen und Blutgefässe gleichzeitig an der Stelle entstehen, wo man sie findet, d. h. innerhalb des Mesoblast. So einig man im Allgemeinen über den Ort der Entstehung des Blutes ist, so gross ist die Uneinigkeit über die Frage, welche von den beiden Blutkörperchenarten zuerst entsteht. Es genüge - da es nicht in unserer Absicht liegt, die schon an anderer Stelle ${ }^{1}$ ) des genaueren besprochenen Ansichten noch einmal zu wiederholen, - darauf hinzuweisen, dass über die Beziehungen der rothen zu den weissen Blutkörperchen die drei möglichen Ansichten herrschen: a) es besteht keinerlei Abhängigkeitsverhältniss zwischen beiden; b) es sind thatsächlich Beziehungen zwischen ihnen vorhanden. Von denen, die der letzteren Ansicht huldigen, behaupten die Einen, - entsprechend der älteren Anschauung, - dass die rothen Blutkörperchen aus den weissen entstehen, während die Anderen die rothen Blutkörperchen als Ursprungszellen der weissen angesehen wissen wollen.

Ausser dieser Frage nach den Beziehungen zwischen den weissen und rothen Blutkörperchen harrt noch eine andere, die besonders in den letzten Jahren die Forscher beschäftigt hat, der Erledigung, ich meine die Frage: Welcher Zusammenhang besteht zwischen den grossen Blutkörperchen der ersten embryonalen Zeit und den bekannten kernhaltigen rothen Blutkörperchen?

1) Engel, Zur Entstehung der körperlichen Elemente des Blutes. Archiv f. mikrosk. Anatomie, Bd. 42. 
Die Beantwortung dieser beiden Fragen hat Verf. zum Gegenstande seiner Untersuchungen gemacht, über welche im Folgenden berichtet werden soll.

Die Untersuchungen wurden in der Weise angestellt, dass Hühnereier bei einer Temperatur von ca. $40^{\circ} \mathrm{C}$. im Brutschrank erwärmt wurden. Nachdem die Eier verschieden lange Zeit bebrutet waren, wurde die Kalkschale an ihrem stumpfen, lufthaltigen Ende vorsichtig entfernt und unter möglichster Schonung der Schalenhaut der Embryo aufgesucht. Die Blutgewinnung geschah in folgender Weise: Da es darauf ankam, das Blut ohne Verunreinigungen durch Eigelb oder Eiweiss und ohne Beimengung von Gewebszellen des Hühnchens zu erlangen, wurde durch die Schalenhaut hindurch mit einem spitzen Scalpell in ein Blutgefäss hineingestochen. Der hervorquellende Blutstropfen wurde theils in ein Capillarröhrchen, theils in einen Capillarraum hineingezogen, der dadurch gebildet wurde, dass zwei Deckgläschen durch eine Pincette aufeinander gedriickt wurden. Das im Capillarröhrchen aufgefangene Blut wurde frisch und in $2 \%$ Osmiumsäure untersucht, das im Capillarraum befindliche Blut wurde nach vorsichtiger Entfernung der beiden Deckgläschen von einander - unter möglichst sorgfältiger Fernhaltung der Finger - lufttrocken werden lassen und entweder in Alcohol absolutus oder auf der Ehrlich'schen Kupferplatte durch vorsichtiges Erhitzen fixirt. Die Osmiumsäure-Präparate wurden theils ungefärbt, theils mit Hämatoxylin gefärbt untersucht, die in Alkohol fixirten Präparate wurden in Hämatoxylin-Eosin, die durch Erhitzen fixirten in Ehrlich's "neutralem Gemisch" gefärbt.

Wir gehen nun zur Schilderung der Blutpräparate tiber und beginnen mit dem 3 Tage alten Hühnerembryonen (Fig. 1).

Die Behandlung des Blutes mit Ehrlich's neutralem Gemisch resp. Eosin-Hämatoxylin ergab:

I. Kugelförmige Zellen mit braunrothem resp. rothem, hämoglobinhaltigem Protoplasma und einem grossen, kreisrunden, graublauen resp. dunkelblauen Kern. Gehen wir auf die Bestandtheile der Zelle etwas specieller ein, so finden wir, dass die Grösse der Zelle zwischen 9 und $20 \mu$ im Durchmesser schwankt. Ausnahmsweise wurde einmal eine Zelle mit 5 Kernen beobachtet, welche einen Durchmesser von $25 \mu$ hatte. Der in Ruhe kreisrunde Kern hat einen Durchmesser von $4-10 \mu$. Er ist durch 
einen farblosen Kreis vom Protoplasma der Zelle getrennt. Dieser Kreis, welcher wahrscheinlieh dadurch entsteht, dass sich beim Trocknen der Zelle der Kern mit seiner Membran in sich zusammenzieht, findet sich niemals bei den zahlreichen Kernen, die Segmentirung zeigen, was als Beweis dafür gelten kann, dass bei der Karyokinese der Kern zu dem umliegenden Protoplasma in viel engerer Beziehung steht als in der Ruhe. Uebrigens zeigen nur die durch Hitze fixirten Präparate diesen farblosen Ring. Auch in anderer Beziehung unterscheidet sich der in Ruhe befindliche Kern von dem segmentirten. Am ruhenden Kern lassen sich durch Färbung mit Ehrlich's neutralem Gemisch deutlich zwei verschieden gefürbte Substanzen (Fig. 1 $a, a^{1}$ ) erkennen: a) eine grünblaue, zuweilen hellblaue in Klümpchen angeordnete Substanz, deren einzelne Theile meist durch Verzweigungen mit einander in Verbindung stehen und welche der chromatischen Substanz angehört und b) eine röthliche, der achromatischen Substanz entsprechende, welche die Fäden und Klümpchen der chromatischen Substanz umgiebt. Die in Karyokinese befindlichen Kerne zeigen ebenfalls eine Doppelfärbung. Hier ist aber von der röthlichen, achromatischen Substanz nichts zu sehen, während jede Schleife der chromatischen Substanz aus einer blau-grïnen Linie besteht, die beiderseits von einer intensiv schwarzen Begrenzungslinie umgeben ist. Diese schwarze Umrandung der Kernfiguir zeigt sich auch noch nach der Theilung in den Kernen, die das Stadium der Ruhe noch nicht erreicht haben.

Ausser diesen Zellen, welche den in der oben erwähnten Arbeit besprochenen Metrocyten (Mutterzellen) entsprechen und auch beim Hubnchen so benannt werden mögen, finden sich aber noch andere Zellformen, von denen jetzt die Rede sein soll.

II. Es sind dies Zellen mit bläulichem, schwach gefärbtem Kern (Fig. 1 $f$ ), der zuweilen ähnliche Structur zeigt, wie der Metrocytenkern. Um den Zellkern, dessen Grösse zwischen 6 und $15 \mu$ schwankt, liegt ein röthlicher meist schmaler Protoplasmasaum herum, sodass die Zelle als weisses Blutkörperchen, und zwar als Lymphocyt anzusehen ist. Diese Lymphocyten liegen oft in Häufchen von 2 bis 10 und mehr Zellen zusammen. Sie haben dann ein gemeinsames Protoplasma, ihre Kerne zeigen in den meisten Fällen versehiedene Grösse. Einige Exemplare haben Kernformen, welche eine Zweitheilung erkennen lassen. 
Karyokinetische Figuren wurden an ihnen nicht gesehen. Sie zeigen aber noch andere Eigenthümlichkeiten. Abgesehen davon, dass ihre Kerne niemals kleiner als die der Metrocyten sind, besitzen oft einzeln liegende Exemplare an ihrer Oberfläche Fortsätze und knotige Verdickungen (Fig. $1 e$ ) von braunrother Farbe, derselben Farbe, wie sie die hämoglobinhaltigen, rothen Blutkörperchen besitzen. Berücksichtigt man ferner, dass einzelne dieser grossen, schwach gefärbten Kerne innerhalb sehr abgeblasster, stark vergrösserter $(16-18 \mu)$ Metrocyten angetroffen werden, so liegt der Schluss nahe, dass diese im Blute des dreitägigen Hühnerembryonen angetroffenen Leukocyten als Kerne von Metrocyten anzusehen sind.

Das Blut des 4. Tages zeigt gegen das des 3. Tages keine Besonderheiten, wir besprechen deshalb das Blut des 5 Tage lang bebrüteten Hühnereies (Fig. 2).

Was zuerst die Metrocyten betrifft, so sind sie zwar in Gestalt, Grösse und Farbe von denen der beiden vorangehenden Tage nicht zu unterscheiden, es ist jedoch auffallend, dass die Kerne etwa nur halb so gross sind, wie die des dritten Tages (Fig. 2a). Die Kerne haben in ihrer Mehrzahl nur noch einen Durchmesser von 4-6 $\mu$. Auch die Zahl der Zellen mit Kerntheilungsfiguren (Fig. 2b) ist eine geringe, doch finden sich häufiger Zellen mit mehreren - bis 4-Kernen in Ruhe. Ausser diesen kleinkernigen Metrocyten, die höchstwahrscheinlich aus den ersteren durch Theilung derselben entstanden sind - weshalb wir für sie den Namen "Metrocyten-Tochterzellen" vorschlagen -, enthält das Blut des 5 Tage alten Hühnerembryonen noch zwei Formen rother Blatkörperchen.

Zunächst kernlose, rothe Blutkörperchen. Diese sind wie die Metrocyten kugelförmig, zuweilen birnförmig (Fig. 2c), haben dieselbe Farbe wie diese, sind jedoch kleiner als die Metrocyten und kommen nur in geringer Anzahl vor.

Ferner enthält das Blut kernhaltige, rothe Blutkörperchen mit kleinem, rundem Kern und geringer Menge hämoglobinhaltigen Protoplasmas, Formen, wie wir sie beim Mensehen „Normoblasten" nennen. Sie unterscheiden sich von den gewöhnlichen kernhaltigen, rothen Blutkörperchen des erwachsenen Hühnchens nur durch die Kleinheit ihrer Protoplasmamasse und ferner dadurch, dass sie rund und nicht elliptisch wie jene sind (Fig. $2 d$ ). 
Ihr Durchmesser beträgt $6-9 \mu$, der Durchmesser ihres Kerns 4-6 $\mu$. Im Gegensatz zu den Metrocyten haben sie ein schwach gefärbtes Protoplasma mit meist zackiger Begrenzung und sehen wie Scheiben aus. Ihr Kern unterscheidet sich durch nichts von dem Kern der Metrocyten-Tochterzellen.

Da wir uns mit den kernlosen rothen Blutkörperchen and den Normoblasten noch später eingehender beschäftigen müssen, so soll hier gleich die Besprechung der übrigen im Blute des 5 Tage alten Hühnerembryonen beobachteten Zellen angeschlossen werden.

Die Lymphocyten (Fig. $2 e$ ) erscheinen als Zellen mit sehr wenig Protoplasma, die die Eigenthümlichkeiten der weissen Blutkörperchen des 3. Tages wiederholen. Von besonderem Interesse ist aber, dass ihre Kerne, entsprechend den kleineren Kernen der Metrocyten-Tochterzellen, kleiner sind als die Kerne der Lymphocyten der vorigen Tage. Nur zuweilen übertrifft ein Lymphocyt des 5. Tages (Fig. $2 e^{2}$ ) einen solchen des 3. Tages an Grösse. Die Lymphkörperchen sind von den kernhaltigen rothen Blutkörperchen nur dadurch zu unterscheiden, dass sie meist in Häufchen zusammensitzen, einen schwächer gefärbten Protoplasmasaum und einen weniger intensiv gefärbten Kern haben (Fig. 2e).

Endlich sind noch die eosinophilen Zellen zu erwähnen (Fig. $2 f$ ), die am 5. Tage zuerst beobachtet werden. Sie sind kleiner als die Eosinophilen des erwachsenen Vogels, haben einen oder zwei schwach gefärbte Kerne und - mit dem neutralen Gemisch $\left.{ }^{1}\right)$ gefärbt - violette theils runde, theils nadelförmige Granula. Sie kommen in sehr geringer Menge vor.

Es muss noch erwähnt werden, dass das Verbältniss der Metrocyten zu den kernhaltigen rothen Blutkörperchen, den Normoblasten, am 5. Tage etwa 20:1 beträgt.

Am 6. Tage zeigt das Blut dasselbe Aussehen wie am 5., nur dass die kernhaltigen, rothen Blutkörperchen, die zum Theil schon Ellipsenform besitzen, an Zahl im Vergleich zu den Metrocyten bedeutend zugenommen haben. Es stellt sich bereits das

1) Auffallend ist, dass die „eosinophilen“ Granulationen des Hühnchens sich nicht mit Eosin fürben liessen. Wir sprechen hier trotzdem von „eosinophilen“ Zellen, weil sie von den $5 \mathrm{Ehrlich}$ 'schen Granulationen den acido- oder eosinophilen am ähnlichsten sind. 
Verhältniss der Metrocyten zu den Normoblasten in den einzelnen Präparaten wie 5 bis $2: 1$. Dabei ist zu bemerken, dass sich die Metrocyten im frisch untersuchten Blute ohne Zuhilfenahme irgend einer Fixirung oder Färbung durch ihre Kugelform - zuweilen Eiform -, ihre Grösse und ihre intensiv gelbe Farbe von den gewöhnlichen, kernhaltigen, rothen Blutkörperchen unterscheiden. Ferner ist es selbstverständlich, dass die Metrocyten in Folge ihrer Kugelform sich nicht geldrollenartig uber einander legen können, was nur bei den scheibenförmigen kernhaltigen rothen Blutkörperchen möglich ist.

Da das Blut am 7. Tage die Eigenthümlichkeiten des achttägigen Blutes, nur in geringerem Maasse, aufweist, so soll jetzt das Blut des 8 Tage bebrüteten Hühnereies etwas genauer besprochen werden. Wir finden hier folgende Formen (Fig. 3):

1. Metrocyten. Sie haben meist Kugelform (Fig. $3 a, a^{1}$ ), selten sind sie ellipsenförmig. Ihr Durchmesser beträgt ca. $12 \mu$, ihr Kern 4-6 $\mu$. Sie haben einen, selten mehrere Kerne. Dieser liegt meist in der Mitte. Sehr auffällige Bilder geben Formen der Metrocyten, bei welchen von der Hauptmasse des intensiv gefärbten Protoplasmas eine protoplasmatische Verlängerung $a b$ geht, in der meistentheils der Kern liegt (Fig. $3 b, b^{1}$ ). Da diese noch nicht gänzlich vollzogene Trennung der Hauptmasse des Protoplasmas von dem eine Protoplasmakrause behaltenden Kern sowohl im frischen Präparat als auch in dem durch Osmiumsäure fixirten in vielen Dutzenden von Exemplaren nachzuweisen ist, so kann von einem Kunstprodukt nicht die Rede sein. In jedem frischen Blutpräparate aus dem 7. bis etwa 16. Tage finden sich solche Trennungsformen.

In demselben Präparate finden sich ferner

2. kernlose rothe Blutkörperchen, welche meistens birnförmig sind und die Stelle erkennen lassen, an der der Kern mit dem an ihm haften bleibenden Protoplasma gesessen hat. Manche kernlose rothe Blutkörperchen haben jedoch die Kugelform angenommen (Fig. $3 d$ ). Ohne Ausnahme zeigen alle diese kernlosen Blutkörperchen die intensive Farbe der Metrocyten. Da jedes kernhaltige rothe Blutkörperchen aus einem Metrocyten hervorgegangen sein muss, so müsste die Zahl der kernlosen die gleiche sein, wie die der kernhaltigen rothen Blutkörperchen. Das ist aber keineswegs der Fall. Während die kernhaltigen 
rothen Blutkörperchen am 8. Tage bereits etwas zahlreicher sind als die Metrocyten, ist die Zahl der Metrocytenreste, d. h. der kernlosen rothen Blutkörperchen sehr gering. Diese gehen also zu Grunde. Dass dies wirklich der Fall ist, geht daraus hervor, dass Fragmente solcher kernloser Blutkörperchen (Fig. $3 d^{1}$ ) schon vom 3. Tage ab während des ganzen embryonalen Lebens zur Beobachtung gelangen. Es bleiben also von den Metrocyten nur die

3. kernhaltigen rothen Blutkörperchen ubrig. Diese haben am 8. Tage zum grossen Theil schon die elliptische Scheibenform (Fig. $3 g^{1}, g^{2}$ etc.) und man sieht im frischen Präparate an sich rollenden Blutkörperchen, wie der Kern die Protoplasmascheibe beiderseits uberragt. Das kernhaltige rothe Blutkörperchen ist also ein flaches Gebilde, welches nicht einmal die Dicke des Kerns erreicht. Entsprechend der Entstehung der kernhaltigen rothen Blutkörperchen lässt ihr Protoplasma sehr häufig noch die Stelle erkennen, an welcher es am Metrocyten gesessen hat. Man sieht häufig an ihnen schrauben- oder schwanzförmige Fortsätze (Fig. $3 g$ ), die nur durch Berticksichtigung ihrer Herkunft erklärt werden können.

Was die tabrigen Zellen in diesem Stadium der Entwickelung des Huhnchens betrifft, so haben auch hier die Lymphocyten dieselbe Form und Grösse, wie im Blute des 5. Tages beschrieben worden ist. Wenn sie einzeln liegen, sind sie nur durch die schwächere Färbung des Kerns und Protoplasmas von den kreisrunden, kernhaltigen rothen Blutkörperchen zu unterscheiden (Fig. $3 e^{1}$ ). In den Fällen, wo ein Metrocyt schon zerfällt, während er den Kern noch besitzt, - was zuweilen vorkommt -, unterscheidet sich sein schwach blau gefärbter Kern von einem Lymphocyten durch nichts. Es muss erwähnt werden, dass einzelne Lymphocyten beobachtet wurden, die sich erstens durch ihre Grösse auszeichneten, besonders aber dadurch auffielen, dass sie einen U- oder E-förmigen Kern zeigten. Von neutrophiler Granulation, wie sie für die polynucleären Zellen der Säugethiere characteristisch ist, war jedoch nichts zu sehen. Es ist also nicht unwabrscheinlich, dass auch hier, wie zuweilen bei grossen Lymphocyten des Menschen zu beobachten ist, der grosse runde Kern Ausbuchtungen bilden kann.

In den Präparaten desselben Alters fanden sich ausserdem 
noch in Zerfall begriffene Kerne zerfallener Metrocyten und endlich sehr wenig eosinophile Zellen mit nadel- oder körnchenförmigen Granulationen.

Es erübrigt, um Wiederholungen zu vermeiden, die weitere Entwickelung des Blutes während der folgenden Tage bis zum Auskriechen des Hühnchens - dem 21. Tage - im Zusammenhange abzuhandeln. Characteristisch für die Entwickelung des Blutes ist stets das Verhältniss der Metrocyten zu den kernhaltigen, rothen Blutkörperchen. War das Verhältniss am 8. Tage etwa wie $1: 1$, so hat es sich schon in den nächsten Tagen bedeutend zu Gunsten der kernhaltigen Rothen geändert. Es kommen etwa am 10. Tage 1 Metrocyt auf 5 bis 10 Normoblasten, am 16. Tage ist das Verhältniss $1: \mathrm{ca} .50$ und von da ab bis zum 21. Tage finden sich nur noch sehr wenige Metrocyten in den Präparaten. Man ist also in der Lage, aus dem Blutbefund anf das Alter des Hühnerembryonen zu schliessen.

Was die kernlosen, rothen Blutkörperchen betrifft, so findet man zwar bis kurz vor dem Auskriechen des Hühnchens hin und wieder eins (Fig. 4 und 5 aus dem 14. und 18. Tage), doch mit dem Verschwinden der Metrocyten haben auch sie ihr Ende erreicht.

Die kernhaltigen, rothen Blutkörperchen, welche ja bekanntlich im Hühnchen bestehen bleiben - während im Säugethierblute die kernlosen Blutkörperchen bestehen bleiben und die kernhaltigen verschwinden -, nehmen von Tag zu Tag mehr ihre normale Form an. Die kreisrunden oder die mit Fortsätzen versehenen werden immer seltener, sodass sich ein Blutpräparat etwa vom 18. Tage von einem Präparate aus dem erwachsenen Hühnchen nur dadurch unterscheidet, dass man beim Durchmustern mehrerer Gesichtsfelder des ersteren Blutes einem oder dem anderen Metrocyten oder einem kernlosen rothen Blutkörperchen begegnet.

Die Leukocyten haben dasselbe Aussehen, wie es am 8. Tage geschildert worden ist. Etwas grösser als die Kerne der kernhaltigen rothen Blutkörperchen sind sie meist in kleinen Gruppen anzutreffen. Auffallend ist, dass zuweilen freie intensiv gefärbte Kerne zu sehen sind, die einen protoplasmatischen Saum vermissen lassen.

Der letzte Bestandtheil des Vogelblutes, die eosinophilen 
Zellen, sind bekanntlich grosse kugelförmige Zellen - von der Grösse der Polyuucleären des Menschen -, die beim Hühnchen nadelförmige Granulationen und einen oder zwei Kerne besitzen. Wir haben oben angegeben, dass die Eosinophilen zuerst am 5. Tage der Bebruitung angetroffen wurden und dass sie an diesem und den nächstfolgenden Tagen meistentheils punktförmige Granulationen hatten. Im weiteren Verlaufe der Entwickelung zeigen sie immer mehr die nadelförmigen Granula, sodass in der dritten Woche der Entwickelung fast nur noch nadelförmige Granulationen angetroffen werden.

Da wir die eosinophilen Zellen nicht in der Entwickelungsreihe von den Metrocyten bis zu den Leukocyten unterbringen konnten und es auch nicht möglich war, irgend eine Beziehung zwischen den weissen Blutkörperchen und den Eosinophilen festzustellen, wurden auch die blutbildenden Organe, also Knochenmark, Milz, Leber - Lymphdrisen herauszupräpariren war nicht möglich --- in den Bereich der Untersuchungen hineingezogen. Die diesbezüglichen Beobachtungen sollen der Gegenstand einer späteren Veröffentlichung sein, hier soll nur erwähnt werden, dass Milz und Knochenmark in Bezug auf die Beantwortung unserer oben formulirten Fragen einen höchst interessanten Aufschluss gaben. In beiden Organen traten 3 Zellformen besonders hervor: 1. Metrocyten, 2. sehr zahlreiche Eosinophile mit runden - sehr selten nadelförmigen Granulationen und 3. grosse Zellen mit fast die ganze Zelle ausfüllendem Kern, um den zuweilen ein hämoglobinhaltiges Protoplasma lag, sodass dann Zellen beobachtet wurden, wie wir sie beim Menschen Megaloblasten zu nennen pflegen. Uns interessiren hier nur die ersten beiden Zellformen. Während das Blut etwa am 18. Tage nur äusserst wenig Metrocyten enthält und fast ganz aus kernhaltigen rothen Blutkörperchen besteht (Fig. 5), finden sich in der Milz an demselben Tage äusserst wenig kernhaltige rothe Blutkörperchen, dagegen eine grosse Anzahl von Metrocyten.

Was die eosinophilen Zellen betrifft, so werden die mit runden Granulis, wie es scheint, fruher gebildet, als die mit nadelförmiger Granulation, und es sind in der Milz die ersteren immer noch sehr zahlreich vorhanden, wenn im Blute fast nur noch Zellen mit nadelförmigen Granulationen vorkommen. 
Weisse Blutkörperchen, einzeln oder in Haufen, wie sie in jedem Blutpräparate zu sehen sind, waren weder in der Milz noch im Knochenmark in besonders grosser Menge vorhanden.

Fassen wir zum Schlusse unsere Beobachtungen zusammen, so ergiebt sich Folgendes:

Die körperlichen Bestandtheile des embryonalen Huihnerblutes sind wesentlich verschieden von denen des erwachsenen Hühnchens. Die ersten Blutkörperchen sind kernhaltige, hämoglobinreiche Zellen, welche mit den kernhaltigen rothen Blutkörperchen des erwachsenen Huhnes nicht identisch sind. Die ersteren sind grösser, kugelförmig, mit einem grossen Kern, der häufig Kernsegmentirung zeigt. Diese Zellen - Metrocyten erster Generation - gehen im Verlauf des 4. bis 5. Tages in die Metrocyten-Tochterzellen oder zweiter Generation uber. Die Metrocyten-Tochterzellen theilen sich nicht mehr durch Karyokinese, haben jedoch zuweilen mehrere Kerne. Vom 5. Tage an tritt eine Zweitheilung des Metrocyten zweiter Generation ein. Der Kern nebst dem ihm zunächst liegenden hämoglobinhaltigen Protoplasma bildet die eine Hälfte, der protoplasmatische, hämoglobinhaltige Rest, die andere. Diese letztere geht zu Grunde, der Kern nebst seiner Protoplasmakrause bildet eine Zelleinheit, welche der "Energide" von Sachs') entspricht. Das aus dem Metrocyten ubrig gebliebene kernhaltige rothe Blutkörperchen nimmt allmählich die Form an, die es im Leben des erwachsenen Thieres hat.

In Betreff der weissen Blutkörperchen lassen sich nicht so sichere Angaben machen. Schon im Blute des drei Tage alten Embryonen finden sich Zellen, die zwar das Aussehen von Lymphkörperchen haben, doch durch Form, Grösse, Kernfärbung und Unregelmässigkeit ihrer Oberfläche eher den Eindruck von Metrocytenkernen machen, deren Protoplasmaleib zu Grunde gegangen ist. Während der späteren Tage ist der Zusammenhąng zwischen Leukocyten und kernhaltigen rothen Blutkörperchen noch auffallender: Nachdem sich der Metrocyt in einen kernhaltigen und

1) Sachs, Vorlesungen über Pflanzenphysiologie, 1882. Mit dem Ausdruck „Energide“ soll der Kern mit dem ihn umgebenden Protoplasma gemeinsam bezeichnet werden. 
einen kernlosen Theil getheilt hat, wächst der kernhaltige Theil entweder - durch Aufnahme von Hämoglobin aus dem Blutplasma, welches ja das Hämoglobin der zu Grunde gegangenen Metrocyten enthalten muss - zu einem definitiven kernhaltigen rothen Blutkörperchen aus, oder er verliert seinerseits sein Hämoglobin, der frei gewordene Kern theilt sich direkt und bildet die als weisse Blutkörperchen anzusprechenden Zellhaufen. Nachdem dieser Zellcomplex sich gelöst hat, wachsen die einzelnen Zellen zu ibrer normalen Grösse aus.

Die eosinophilen Zellen endlich haben mit den Metrocyten nichts zu thun, sie kommen nach der Bildung der Blutbildungsorgane - also etwa dem 5. Tage - in die Blutbahn und wandeln auf dem Wege ins Blut ihre punktförmigen Granula in nadelförmige um. Zusammen mit den unentwickelten eosinophilen Zellen kommen höchstwahrscheinlich neugebildete Metrocyten aus den Blutbildungsorganen in die Blutbahn, wo sie sich dann weiter entwickeln.

Vergleichen wir noch für einen Angenblick die Blutentwickelung beim Säugethier mit der beim Hühnchen, so ergiebt sich, dass das Blut beider Thierklassen vom Metrocyten bis zum kernhaltigen rothen Blutkörperchen dieselben Stadien durchläuft, dass dann das Huhnerblut auf diesem Stadium stehen bleibt, während das Blut des Säugethiers nur in pathologischen Füllen mehr oder weniger auf dieser Entwicklungsstufe behart, sich aber normalerweise weiter entwickelt.

Bevor ich die Arbeit beende, erfulle ich die angenehme Pflicht, Herrn Prof. 0. Hertwig für das Interesse, welches derselbe meinen Untersuchungen entgegen gebracht hat, meinen herzlichsten Dank auszusprechen. 


\section{Erklärung der Abbildungen auf Tafel XVII.}

Die Vergrösserung beträgt $1: 1500$.

Fig. 1. Blut eines 3 Tage alten Hühnerembryonen.

$a, a^{1}=$ Metrocyten erster Generation mit einem und zwei Kernen in Ruhe.

$b \quad=$ Kerntheilungsfigur.

$c \quad=$ Theilung in zwei Metrocyten-Tochterzellen.

$d, d^{1}=$ Metrocyten-Tochterzellen gleich nach der Theilung.

$e=$ Metrocyten-Kern mit Protoplasmaresten.

$f, f^{1}=$ Leukocyten.

Fig. 2. Blut eines 5 Tage alten Embryonen.

$a, a^{1}$ etc. $=$ Metrocyten-Tochterzellen in Ruhe.

$b \quad=$ Metrocyt in Theilung.

c $\quad=$ Metrocyten-Rest, kernloses rothes Blutkörperchen.

$d \quad=$ Kernhaltiges rothes Blutkörperchen.

$e, e^{1}, e^{2}=$ Lymphocyten.

$f \quad=$ Eosinophile Zelle.

$a, a^{\mathbf{1}}=$ Metrocyten.

Fig. 3. Blut vom 8. Tage.

$b, b^{1}=$ Loslösung des kernhaltigen rothen Blutkörperchens vom Metrocyten.

$c \quad=$ frei gewordenes kernhaltiges rothes Blutkörperchen.

$d, d^{1}=$ kernlose rothe Blutkörperchen.

$e \quad=$ Lymphocyt, fast identisch mit einem Metrocytenkern.

$f \quad=$ Lymphocytenhaufen.

$g, g^{1}$ etc. = kernhaltige rothe Blutkörperchen.

Fig. 4. Blut vom 14. Tage, Eosin Hämatoxylin.

$a$ = Metrocyt.

$b \quad=$ Trennung des Metrocyten in einen kernhaltigen und einen kernlosen Theil.

c $=$ kernhaltiges, rothes Blutkörperchen, gleich nach der Trennung.

$d, d^{1}, d^{2}, d^{3}=$ Lymphocyten, einzeln und in Häufchen.

$e \quad=$ gewöhnliche rothe Blutkörperchen.

Fig. 5. Blut vom 18 Tage.

$a=$ Metrocyt.

$b=$ kernloses rothes Blutkörperchen.

$c=$ freier Kern.

$d=$ Lymphocytenhaufen.

$e=$ gewöhnliche rothe Blutkörperchen.

$f^{\prime}=$ Eosinophile Zelle. 

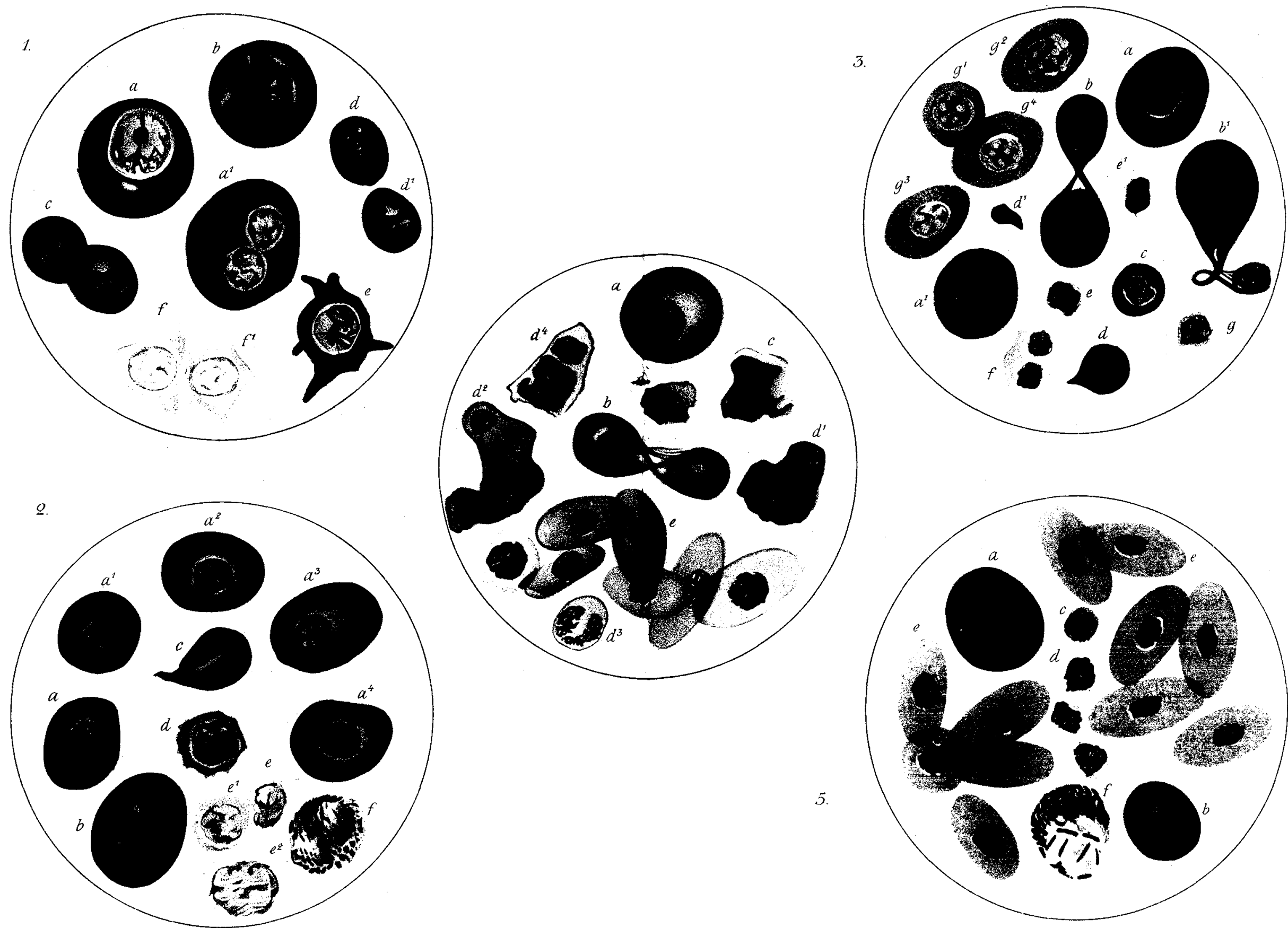